\title{
Visible-light-driven photocatalytic hydrogen production coupled with selective oxidation of benzyl alcohol over CdS@MoS 2 heterostructures
}

\author{
Peixian $\mathrm{Li}^{1,2,3,4}$, Hui Zhao ${ }^{1,3,4}$, Xuyan Yan ${ }^{1,2,3,4}$, Xue Yang ${ }^{1,3,4}$, Jingjun $\mathrm{Li}^{1,3,4}$, Shuiying Gao ${ }^{1,3,4^{*}}$ and \\ Rong Cao ${ }^{1,3,4^{*}}$
}

\begin{abstract}
Photocatalytic hydrogen production coupled with selective oxidation of organic substrates to produce highvalue-added fine chemicals has drawn increasing attention. Herein, we report a noble metal-free photocatalyst for the highly efficient and simultaneous generation of hydrogen and the selective oxidation of benzyl alcohol into benzaldehyde over $\mathrm{CdS} @ \mathrm{MoS}_{2}$ heterostructures under visible light. Without the need for a sacrificial agent, $\mathrm{CdS} @ \mathrm{MoS}_{2}$ displayed an excellent hydrogen production rate of $4233 \mu \mathrm{mol} \mathrm{g} \mathrm{g}^{-1} \mathrm{~h}^{-1}$ with $0.3 \mathrm{mmol}$ benzyl alcohol, which is approximately 53 times higher than that of bare CdS nanorods $\left(80 \mu \mathrm{mol} \mathrm{g}^{-1} \mathrm{~h}^{-1}\right)$. The reaction system was highly selective for the oxidation of benzyl alcohol into benzaldehyde. When the amount of benzyl alcohol increased to $1.0 \mathrm{mmol}$, the hydrogen production reached $9033 \mu \mathrm{mol} \mathrm{g}{ }^{-1} h^{-1}$. Scanning electron microscopy and transmission electron microscopy images revealed that p-type $\mathrm{MoS}_{2}$ sheets with a flower-like structure closely adhered to n-type semiconductor CdS nanorods through the formation of a p-n heterojunction. As a potential Z-scheme photocatalyst, the $\mathrm{CdS} @ \mathrm{MoS}_{2}$ heterostructure effectively produces and separates electron-hole pairs under visible light. Thus, the electrons are used for reduction to generate hydrogen, and the holes oxidize benzyl alcohol into benzaldehyde. Moreover, a mechanism of photogenerated charge transfer and separation was proposed and verified by photoluminescence, electrochemical impedance spectroscopy, photocurrent and Mott-Schottky measurements. The results reveal that the $\mathrm{CdS} @ \mathrm{MoS}_{2}$ heterojunctions have rapid and efficient charge separation and transfer, thereby greatly improving benzyl alcohol dehy-
\end{abstract}

drogenation. This work provides insight into the rational design of high-performance Z-scheme photocatalysts and the use of holes and electrons to obtain two valuable chemicals simultaneously.

Keywords: photocatalysis, CdS, $\mathrm{MoS}_{2}$, hydrogen production, benzyl alcohol

\section{INTRODUCTION}

Photocatalytic water splitting to achieve clean hydrogen energy has become a promising strategy for meeting energy demand and addressing environmental challenges [1-9]. Unfortunately, most reports on the process of photocatalytic water splitting have focused on the halfreaction of consuming holes by applying various sacrificial agents such as triethanolamine, triethylamine and $\mathrm{CH}_{3} \mathrm{OH}$ to achieve high hydrogen production. In most cases, the oxidation half-reaction has suffered from sluggish kinetics and limited the overall photocatalytic process [10]. To overcome these limitations, taking advantage of the oxidative ability of holes and using organic substances to displace the toxic and expensive sacrificial electron donors is a promising method, because the holes can oxidize organic substrates into value-added products $[10,11]$. The selective oxidation of alcohols is recognized as an important organic reaction, and the carbonyl derivatives have been industrialized and widely used in laboratory synthesis [12-14]. It remains a challenge to realize highly efficient simultaneous hydrogen production

\footnotetext{
${ }^{1}$ State Key Laboratory of Structural Chemistry, Fujian Institute of Research on the Structure of Matter, Chinese Academy of Sciences, Fuzhou 350002, China

${ }^{2}$ College of Chemistry and Materials Science, Fujian Normal University, Fuzhou 350007, China

${ }^{3}$ University of the Chinese Academy of Sciences, Beijing 100049, China

${ }^{4}$ Fujian College, University of Chinese Academy of Sciences, Fuzhou 350002, China

* Corresponding authors (emails: rcao@fjirsm.ac.cn (Cao R); gaosy@fjirsm.ac.cn (Gao S))
} 
and benzyl alcohol oxidation using holes directly under anaerobic conditions without applying sacrificial agents [15]. For example, Chen and coworkers [15] reported the photocatalytic selective oxidation of benzyl alcohol to benzaldehyde via direct hole oxidation and hydrogen production using the $\mathrm{Pt} / \mathrm{Zn}_{3} \mathrm{In}_{2} \mathrm{~S}_{6}$ hybrid photocatalytic system. Taking advantage of the oxidative ability of holes, $\mathrm{Du}$ and coworkers [16] described a strategy for the highly efficient and simultaneous hydrogen evolution and benzaldehyde production using cadmium sulfide (CdS) nanorods decorated with small cobalt nanoparticles under visible light.

Semiconductor catalysts, such as $\mathrm{TiO}_{2}$ [16], $\mathrm{ZnS}$ [17], and CdS $[18,19]$, have been used for photocatalytic hydrogen production owing to their low cost, good stability, and low toxicity. CdS is particularly amenable for visible-light-driven hydrogen production because of its narrow bandgap and suitable position of the band edge [20-22]. However, CdS has some disadvantages, such as the absence of a catalytically active site and a rapid recombination rate of the photogenerated charge carriers [23-25]. To avoid the above-mentioned problems, constructing heterostructures is an attractive way to effectively promote the rate of charge separation and expose more active sites, thereby improving the photocatalytic activity of CdS [26,27]. As a type of heterostructure, $\mathrm{p}-\mathrm{n}$ heterojunctions are often constructed through staggered energy levels, which gives rise its special optical, electrical, and optoelectronic properties $[28,29]$. Since the $p-n$ junction forms an internal space charge region, the free holes in the $\mathrm{p}$-region and the free electrons in the n-region migrate to the opposite region, which greatly promotes the charge transport and improves the catalytic activity [28]. In most p-type materials, molybdenum disulfide $\left(\mathrm{MoS}_{2}\right)$ is prominent owing to its narrow bandgap, cost-effectiveness and noble metal-free nature [30-32]. Moreover, because the semiconductor CdS exhibits n-type properties and intersects with the energy level of p-type $\mathrm{MoS}_{2}$, a p-n junction is likely to be formed between them, which would also contribute to enhanced photocatalytic activity [33-35]. Regarding the charge transfer mechanism, the Z-scheme is a more promising transfer mechanism because it can greatly shorten the charge transfer route and effectively separate carriers [36,37].

Herein, we demonstrate the extremely efficient, visiblelight-driven, simultaneous photocatalytic production of hydrogen with the selective oxidation of benzyl alcohol into benzaldehyde. The process has high selectivity (99\%) and is carried out over CdS@MoS ${ }_{2}$ heterostructures under visible light $(\lambda>420 \mathrm{~nm})$. The obtained hydrogen activity is 53 times higher than that of bare CdS nanorods. The reasons for the highly efficient production of hydrogen and benzyl alcohol oxidation mainly include the formation of heterojunctions between $\mathrm{MoS}_{2}$ and CdS, exposing more active sites, and adopting a Z-scheme transfer mechanism to increase the charge transport rate and inhibit the undesired electron-hole recombination. The design of reasonable noble metal-free heterojunction photocatalysts provides a sustainable development strategy in the application of photocatalytic hydrogen production and benzyl alcohol oxidation to meet the growing global energy demand.

\section{EXPERIMENTAL SECTION}

\section{Materials}

L-cysteine $\left(\mathrm{C}_{3} \mathrm{H}_{7} \mathrm{NO}_{2} \mathrm{~S}\right.$, Adamas), tetraethylenepentamine (TEPA, Acros), and benzyl alcohol $\left(\mathrm{C}_{7} \mathrm{H}_{8} \mathrm{O}\right.$, Aladdin) were acquired from the respective suppliers. Cadmium nitrate $\left(\mathrm{Cd}(\mathrm{AC})_{2} \cdot 2 \mathrm{H}_{2} \mathrm{O}\right), \quad D(+)$-glucose, sodium molybdate $\left(\mathrm{Na}_{2} \mathrm{MoO}_{4} \cdot 2 \mathrm{H}_{2} \mathrm{O}\right)$, and thioacetamide $\left(\mathrm{C}_{2} \mathrm{H}_{5} \mathrm{NS}\right)$ were purchased from Sinopharm Chemical Reagent Co., Ltd. All reagents were used without further purification.

\section{Synthesis of CdS nanorods}

CdS nanorods were synthesized by a solvothermal method according to a previous report [38]. First, $2 \mathrm{mmol}$ of $\mathrm{Cd}(\mathrm{AC})_{2} \cdot 2 \mathrm{H}_{2} \mathrm{O}$ and $4 \mathrm{mmol}$ of $\mathrm{C}_{3} \mathrm{H}_{7} \mathrm{NO}_{2} \mathrm{~S}$ were added into a Teflon-lined autoclave container containing $50 \mathrm{~mL}$ of TEPA. The autoclave was sealed and maintained at $180^{\circ} \mathrm{C}$ for $24 \mathrm{~h}$. After naturally cooling to room temperature, the yellow precipitate was collected by centrifugation and washed three times with deionized water and absolute ethanol. The final products were dried at $70^{\circ} \mathrm{C}$ under vacuum for $24 \mathrm{~h}$.

\section{Synthesis of CdS@MoS ${ }_{2}$ photocatalyst}

Typically, $0.1 \mathrm{~g}$ of the as-prepared CdS nanorods was added to $20 \mathrm{~mL}$ of deionized water and sonicated for $5 \mathrm{~min}$. Next, $0.2 \mathrm{mmol}$ of glucose, $0.1 \mathrm{mmol}$ of $\mathrm{Na}_{2} \mathrm{MoO}_{4} \cdot 2 \mathrm{H}_{2} \mathrm{O}$ and $0.8 \mathrm{mmol}$ of thioacetamide were added to the suspension. Thioacetamide provides $\mathrm{S}$ atoms for the formation of $\mathrm{MoS}_{2}$ [39], whereas glucose helps to synthesize $\mathrm{MoS}_{2}$ sheets with a flower-like structure and serves as a binder to help $\mathrm{MoS}_{2}$ grow along the longitudinal axis of the CdS nanorods. The mixture was stirred for $20 \mathrm{~min}$ at room temperature. Afterward, the suspension was transferred into a $40-\mathrm{mL}$ autoclave container and heated at $200^{\circ} \mathrm{C}$ for $24 \mathrm{~h}$. The prepared pro- 
ducts were washed with ethanol several times and dried in a vacuum oven. Pure $\mathrm{MoS}_{2}$ with a flower-like structure was also obtained under the consistent conditions in the absence of CdS.

\section{Electrochemical measurements}

To prepare the working electrode, $5 \mathrm{mg}$ of catalyst powder was mixed with $0.7 \mathrm{~mL}$ of $\mathrm{DMF}$ and $0.3 \mathrm{~mL}$ of ethanol and sonicated for a certain period of time. For the electrochemical measurements, the suspension $(40 \mu \mathrm{L})$ was dip-coated onto a fluorine-doped tin oxide (FTO) glass electrode that had a catalyst-coated area of $1.0 \mathrm{~cm}^{2}$. All the electrochemical measurements were carried out on a Zahner Instruments electrochemical station in a typical three-electrode setup with $0.2 \mathrm{~mol} \mathrm{~L}^{-1} \mathrm{Na}_{2} \mathrm{SO}_{4}$ as the electrolyte, $\mathrm{Ag} / \mathrm{AgCl}$ was used as the reference electrode, Pt plate as the counter electrode, and the catalystcoated FTO glass as the working electrode. In addition, the light irradiation source was consistent with the conditions used in the photoactivity tests of this study.

\section{Photocatalytic reactions}

The photocatalytic dehydrogenation of benzyl alcohol was carried out in a 200-mL custom-made glass with a quartz window. In a typical photocatalytic test, $10 \mathrm{mg}$ of photocatalyst was dispersed in $9 \mathrm{~mL}$ of anhydrous acetonitrile containing $0.3 \mathrm{mmol}$ of benzyl alcohol. A 300-W Xe lamp equipped with a cutoff filter $(\lambda>420 \mathrm{~nm})$ was used for visible light irradiation. Before irradiation, the reaction system was purged at least three times with highpurity argon to completely remove the air inside and to ensure the system was in an anaerobic condition. The hydrogen gas product was detected and quantified by an Agilent 7820A gas chromatography (GC) system. After being irradiated for a certain period of time, the mixture was filtered to remove the solid photocatalyst. Next, $100 \mu \mathrm{L}$ of the supernatant liquid containing benzyl alcohol and its oxidation product was diluted with ethyl alcohol and injected into the GC system (Agilent 7890A) equipped with a flame ionization detector (FID). When the photocatalytic reaction was finished, the photocatalyst was collected by centrifugation and washed with ethanol three times. Finally, the collected photocatalyst was dried at $70^{\circ} \mathrm{C}$ and reused for a subsequent catalytic cycle.

The conversion of benzyl alcohol and the selectivity of benzaldehyde were defined as follows:

Conversion $(\%)=\left[\left(C_{0}-C_{\text {benzyl alcohol }}\right) / C_{0}\right] \times 100 \%$,

Selectivity $(\%)=\left[C_{\text {benzaldehyde }} /\left(C_{0}-C_{\text {benzyl alcohol }}\right)\right] \times 100 \%$,

where $C_{0}$ is the initial concentration of benzyl alcohol,
$C_{\text {benzyl alcohol }}$ and $C_{\text {benzaldehyde }}$ are the concentrations of the substrate benzyl alcohol and benzaldehyde.

\section{RESULTS AND DISCUSSION}

\section{Photocatalytic activity of the CdS@MoS 2 photocatalyst}

As shown in Scheme 1, under the excitation of visible light, the CdS@MoS $\mathrm{M}_{2}$ catalyst generates electron-hole pairs, where the electrons migrate to the position of the conduction band (CB), leaving holes in the position of the valence band (VB). Subsequently, benzyl alcohol is oxidized into benzaldehyde by the holes through dehydrogenation, and the two released $\mathrm{H}^{+}$ions are reduced into $\mathrm{H}_{2}$ by the electrons. To make full use of the electrons and holes and obtain two valuable chemicals, this study investigated the photocatalytic dehydrogenation of benzyl alcohol. Fig. 1a shows the photocatalytic properties of benzyl alcohol dehydrogenation using different catalysts under the same conditions. $\mathrm{No}_{2}$ or benzaldehyde was detected in the absence of photocatalyst. $\mathrm{MoS}_{2}$ also showed no activity. With bare CdS nanorods, no benzaldehyde was detected and only a small amount of hydrogen was generated $\left(80 \mu \mathrm{mol} \mathrm{g}^{-1} \mathrm{~h}^{-1}\right)$. Within $3 \mathrm{~h}$, the benzaldehyde yield reached $42 \%$ with the CdS@MoS composite. After 3-h irradiation, the hydrogen production rate of the CdS@MoS 2 catalyst was $4233 \mu \mathrm{mol} \mathrm{g}{ }^{-1} \mathrm{~h}^{-1}$, which is 53 times higher than that of the bare CdS nanorods. In comparison, for the mechanical mixture of $\mathrm{CdS} / \mathrm{MoS}_{2}$, the production of hydrogen and benzaldehyde was only 206 and $369 \mu \mathrm{mol} \mathrm{g}^{-1} \mathrm{~h}^{-1}$, respectively, which was far less than the activity of the CdS@MoS 2 heterostructure. The photocatalytic activity of $\mathrm{CdS} @ \mathrm{MoS}_{2}$ was significantly improved due to the p-type $\mathrm{MoS}_{2}$ tightly

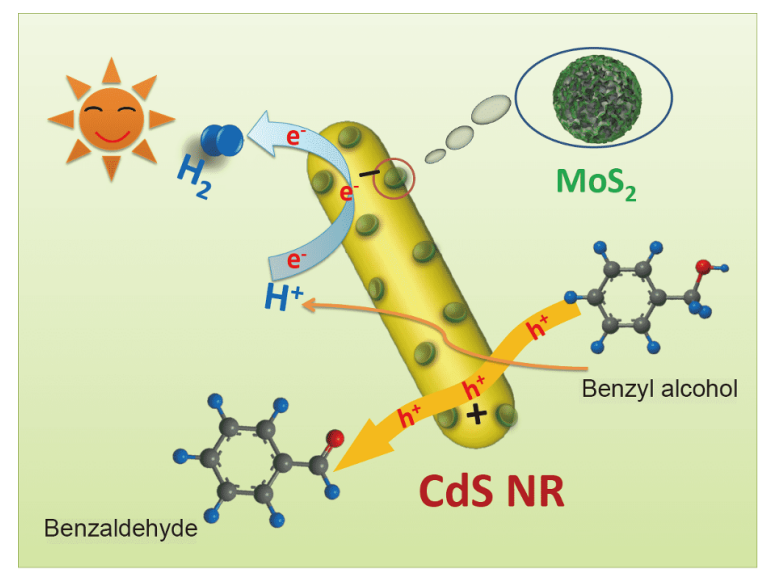

Scheme 1 Photocatalytic dehydrogenation of benzyl alcohol over $\mathrm{CdS} @ \mathrm{MoS}_{2}$ heterostructures under visible light irradiation. 

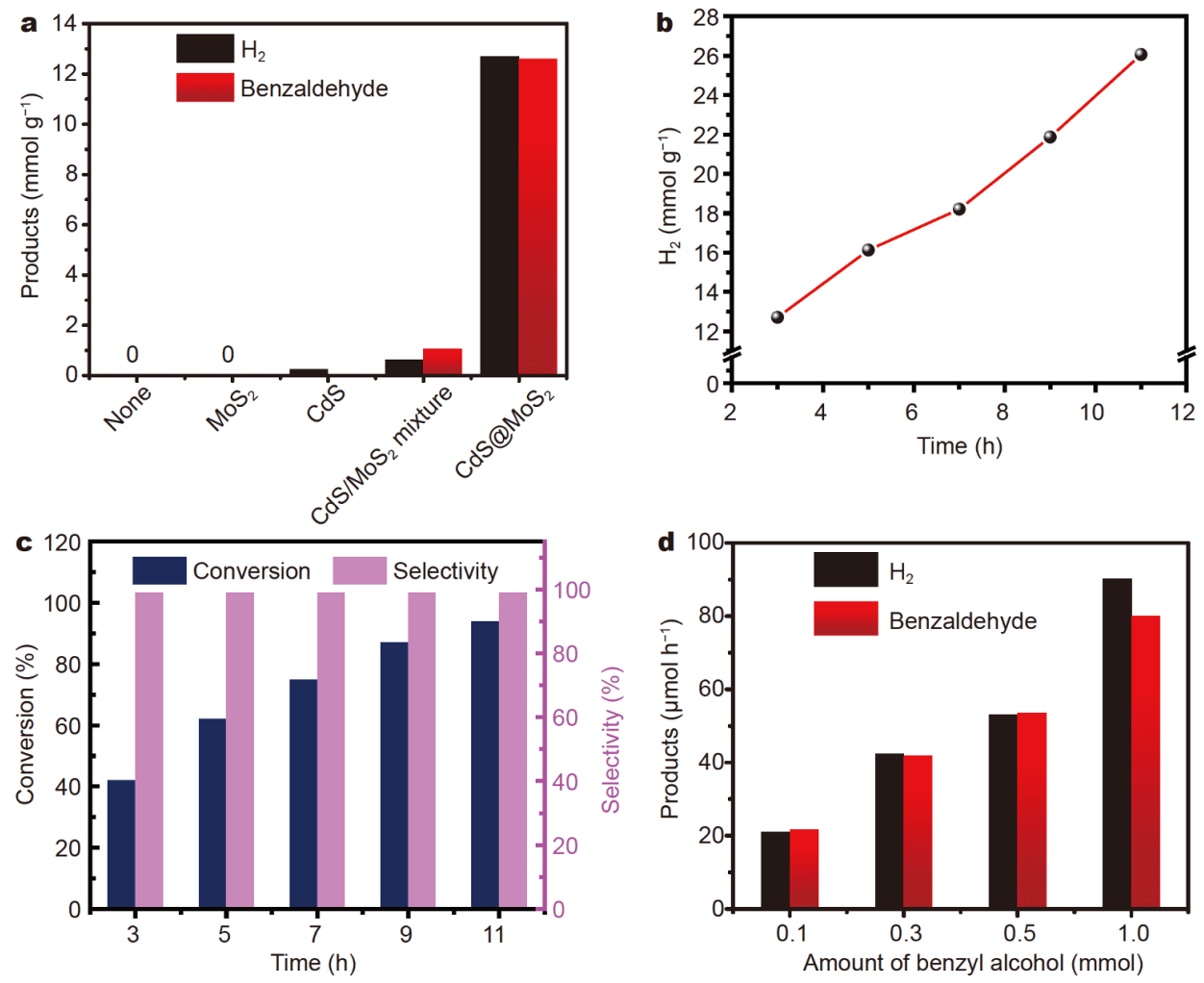

Figure 1 (a) Photocatalytic properties of benzyl alcohol dehydrogenation using different catalysts. Reaction conditions: $\mathrm{CH}_{3} \mathrm{CN}$ : $9 \mathrm{~mL}$, benzyl alcohol: $0.3 \mathrm{mmol}$, room temperature, time: $3 \mathrm{~h}$. (b) Hydrogen production and (c) oxidation of benzyl alcohol over time under visible light $(\lambda>420 \mathrm{~nm})$ using CdS@MoS ${ }_{2}$. (d) Photocatalytic activities of CdS@MoS 2 with different amounts of benzyl alcohol.

adhered to the CdS nanorods and the formation of the p-n junction heterostructure.

Fig. $1 b$ and $c$ show that the rate of $\mathrm{H}_{2}$ production and the conversion rate of benzyl alcohol increased with irradiation time under visible light. Moreover, the selectivity for benzaldehyde was 99\%. After 11-h irradiation, $26 \mathrm{mmol} \mathrm{g}^{-1}$ of hydrogen was produced, whereas the conversion rate of benzyl alcohol reached $94 \%$. To clarify the source of hydrogen protons, comparative photocatalytic experiments were carried out without benzyl alcohol and only adding $9 \mathrm{~mL}$ of acetonitrile or replacing the benzyl alcohol with the same volume $(30 \mu \mathrm{L})$ of other sacrificial agents (such as methanol and triethanolamine) under the same conditions. The results showed that no hydrogen was detected, suggesting the generation of hydrogen was from the dehydrogenation of benzyl alcohol. To confirm the simultaneous generation of hydrogen and benzaldehyde in the CdS@MoS $\mathrm{M}_{2}$ system, the photocatalytic dehydrogenation of benzyl alcohol was further investigated using different amounts of benzyl alcohol. As shown in Fig. 1d, both hydrogen and benzaldehyde were produced simultaneously, and their yields increased with the amount of benzyl alcohol. Specifically, when the amount of benzyl alcohol was $0.3 \mathrm{mmol}, 127 \mu \mathrm{mol}$ of hydrogen and $126 \mu \mathrm{mol}$ of benzaldehyde were simultaneously produced after 3-h of irradiation. This indicates that the amount of the produced hydrogen exactly corresponds to the number of hydrogen protons removed by benzyl alcohol. When the amount of benzyl alcohol was increased to $1.0 \mathrm{mmol}$, hydrogen and benzaldehyde production were respectively increased to 271 and $240 \mu \mathrm{mol}$, and the ratio of hydrogen to benzaldehyde production was 1.1:1. Because no other carbonyl compounds were detected by GC, we speculate that a small amount of benzaldehyde produced under high concentrations of benzyl alcohol is adsorbed onto the catalyst, implying that the actual measured yield is low. Therefore, the ratio of hydrogen to benzaldehyde production was not 1:1. The CdS@MoS heterostructure demonstrated an excellent hydrogen production rate of $9033 \mu \mathrm{mol} \mathrm{g} \mathrm{g}^{-1}$ with $1.0 \mathrm{mmol}$ of benzyl alcohol. Compared with previous reports $[15,40-$ 44] (Table S1), CdS@MoS 2 has a relatively high photocatalytic activity, which is mainly due to the formation of 
Z-scheme heterojunctions and a rapid charge carrier transfer.

Recycling tests were carried out to explore the stability

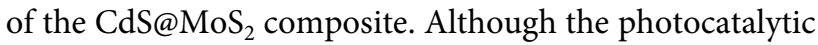
activity of the CdS@ $\mathrm{MoS}_{2}$ photocatalyst decreased after five cycles, it was still much higher than that of bare CdS nanorods (Fig. 2a). The X-ray diffraction (XRD) patterns (Fig. 2b) of the CdS@MoS 2 catalyst were nearly identical after the catalytic reaction, indicating its excellent recyclability. Furthermore, the Cd 3d, Mo $3 d$ and S 2p in the fresh and used CdS@MoS 2 photocatalysts had similar binding energy peaks in the X-ray photoelectron spectroscopy (XPS) spectra without significant shifts, which further indicates the stability of the catalyst (Fig. S1).

\section{Characterizations of the CdS@MoS 2 photocatalyst}

The CdS@ $\mathrm{MoS}_{2}$ heterojunction was synthesized by a simple hydrothermal method. Fig. 3 presents the XRD patterns of the CdS nanorods, pure $\mathrm{MoS}_{2}$ and $\mathrm{CdS} @ \mathrm{MoS}_{2}$ composite. The XRD pattern of $\mathrm{CdS}$ nanorods is consistent with JCPDS card no. 41-1049 of CdS. An obvious small diffraction peak appears at approximately $10.0^{\circ}$ corresponding to (002) of $\mathrm{MoS}_{2}$, confirming the successful adhesion of $\mathrm{MoS}_{2}$ onto the CdS nanorods.
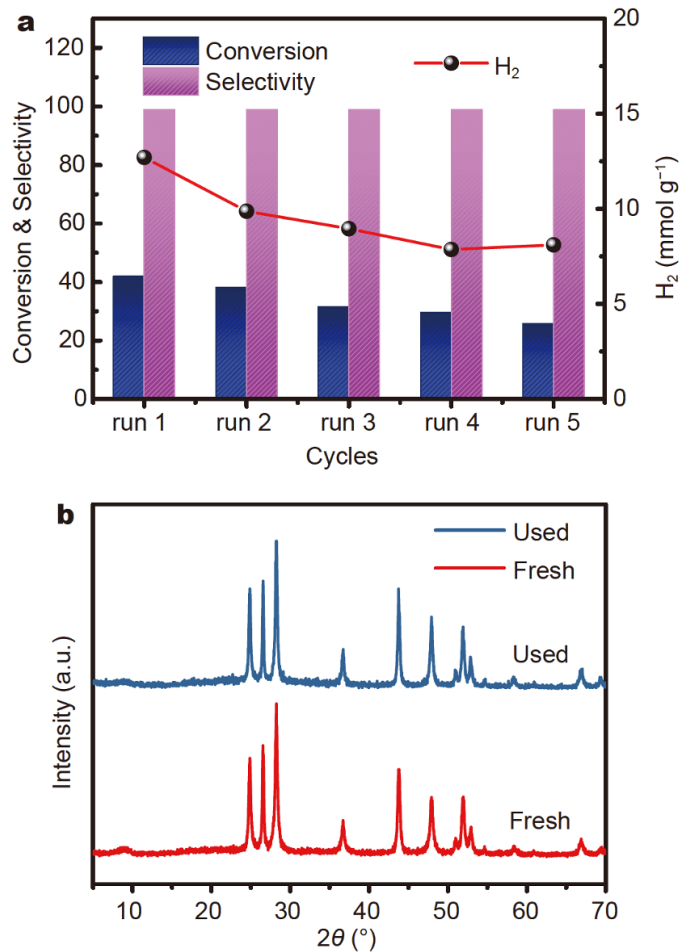

Figure 2 (a) Recycling test of $\mathrm{CdS} @ \mathrm{MoS}_{2}$ for simultaneous hydrogen production and benzyl alcohol oxidation under visible light irradiation for $3 \mathrm{~h}$. (b) XRD patterns of the recycled photocatalyst.
Moreover, using inductively coupled plasma (ICP), we measured the Mo content to be $9.59 \%$ in the CdS@MoS composite, which clearly proved the successful loading of the $\mathrm{MoS}_{2}$. In addition, the successful synthesis of $\mathrm{MoS}_{2}$ was confirmed by the XRD patterns that correspond to published patterns, although there was a shift in the diffraction peak at $2 \theta=14.5^{\circ}-10.0^{\circ}$ compared with JCPDS no. 37-1492 [45-49].

Scanning electron microscopy (SEM) and transmission electron microscopy (TEM) were used to investigate the morphology and structure of the CdS@MoS ${ }_{2}$ composite. Fig. S2 shows the uniform CdS nanorods. $\mathrm{MoS}_{2}$ has a spherical flower-like structure. As shown in Fig. S3, MoS formed many sheet-like structures curled up and stacked together, further forming a flower-like structure. The SEM images show that the $\mathrm{MoS}_{2}$ clearly adhered to the $\mathrm{CdS}$ nanorods (Fig. 4a and b), which explains the for-

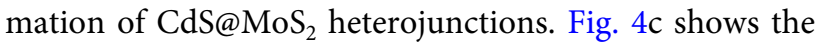
TEM image of CdS@MoS , which clearly illustrates that the $\mathrm{MoS}_{2}$ sheets were well-dispersed onto the CdS nanorods. Moreover, a high-resolution TEM (HRTEM) image of the CdS@MoS 2 composite is presented in Fig. $4 \mathrm{~d}$, which confirms the presence of two distinctly different lattice fringes at 0.337 and $0.271 \mathrm{~nm}$, corresponding to CdS and $\mathrm{MoS}_{2}$, respectively.

XPS was performed to analyze the elemental composition and valence state of the CdS@MoS , photocatalyst. Fig. 5a shows the XPS survey scan of the CdS@MoS composite, which contains $\mathrm{Cd}, \mathrm{Mo}$, and S elements. The high-resolution XPS spectrum of $\mathrm{Cd}$ exhibited two different peaks at the binding energies of 405.4 and $412.1 \mathrm{eV}$ (Fig. 5b), which correspond to $\mathrm{Cd} 3 \mathrm{~d}_{3 / 2}$ and $\mathrm{Cd} 3 \mathrm{~d}_{5 / 2}$ of $\mathrm{Cd}^{2+}$ in CdS, respectively [50]. As shown in Fig. 5c, the Mo 3d XPS spectrum presented doublet peaks at 228.5

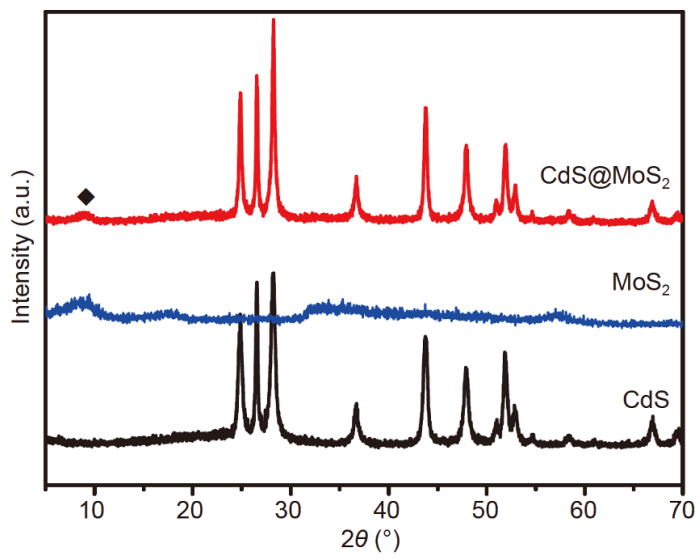

Figure 3 XRD patterns of CdS NRs, pure $\mathrm{MoS}_{2}$ and $\mathrm{CdS} @ \mathrm{MoS}_{2}$ composite. 


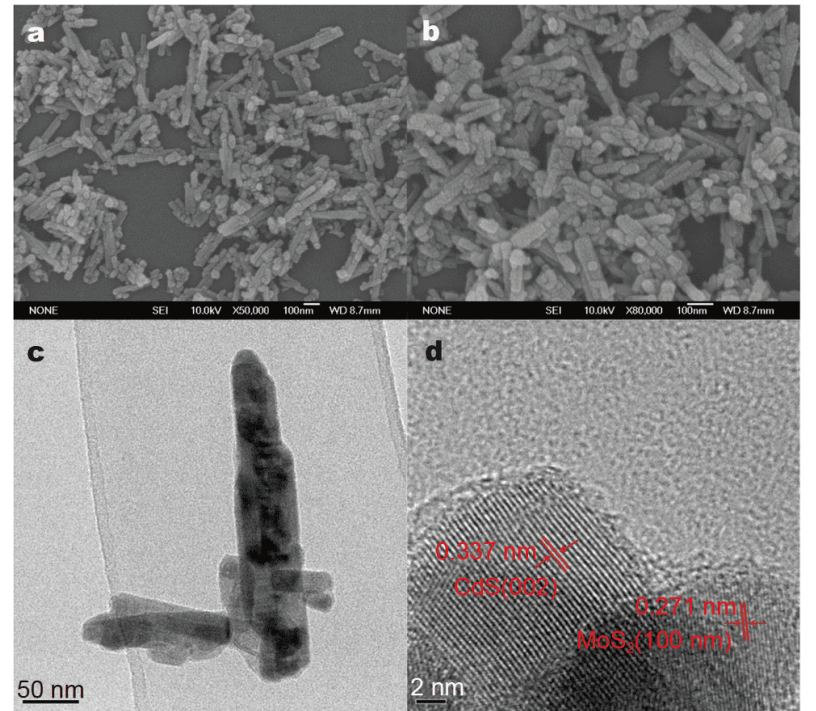

Figure 4 The morphology of the CdS@MoS ${ }_{2}$ composite. (a, b) SEM, (c) TEM, and (d) HRTEM images.

and $231.9 \mathrm{eV}$, respectively, assigned to the Mo $3 \mathrm{~d}_{5 / 2}$ and $3 \mathrm{~d}_{3 / 2}$ of $\mathrm{Mo}^{4+}$ in $\mathrm{MoS}_{2}$ [51]. The strong binding energy at $226.0 \mathrm{eV}$ matches well with the $\mathrm{S} 2 \mathrm{~s}$ bands in sulfides [52]. A small shoulder peak at $234.6 \mathrm{eV}$ was also observed, indicating the existence of $\mathrm{Mo}^{6+}$, probably because the raw materials were not fully reduced $[33,53]$. Moreover, the high-resolution XPS spectrum of $S 2 p$ showed two distinct peaks at binding energies of 161.6 and $162.9 \mathrm{eV}$ (Fig. 5d), which are respectively assigned to the $S 2 p_{3 / 2}$ and S $2 \mathrm{p}_{1 / 2}$ in the form of $\mathrm{S}^{2-}$ in the CdS@MoS 2 composite [50].

\section{Mechanism of hydrogen production and benzyl alcohol oxidation}

Photoluminescence (PL) spectroscopy was carried out to analyze the electron-hole pair recombination of CdS@ $\mathrm{MoS}_{2}$ (Fig. 6a). The PL spectral intensity of $\mathrm{CdS} @ \mathrm{MoS}_{2}$ was lower than that of the bare CdS nanorods at an excitation wavelength of $365 \mathrm{~nm}$, which illustrated that the composite had a lower electron-hole recombination rate and faster charge separation efficiency. Photoelectrochemical experiments were performed to further study the recombination and separation efficiency of the electron-hole pairs. The electrochemical impedance spectroscopy (EIS) results are shown in Fig. 6b, and the arc radius of the EIS Nyquist plot of the CdS@MoS composite was smaller than that of bare CdS nanorods. It is generally believed that the smaller the arc radius, the faster the interface charge transfer [54,55]. For this reason, a faster interfacial charge transfer occurs on the
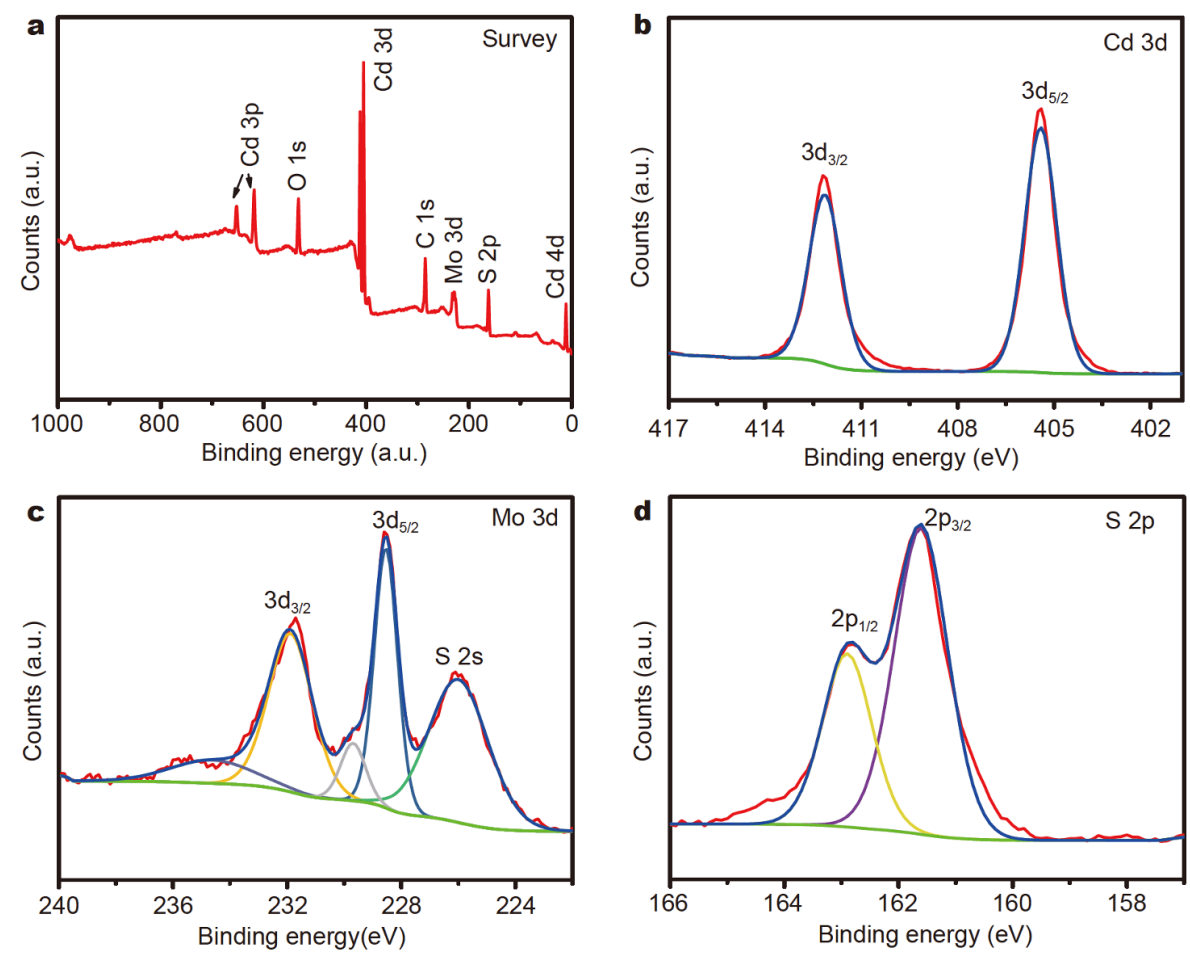

Figure 5 XPS spectra of the CdS@MoS 2 composite. (a) survey, (b) Cd 3d, (c) Mo 3d and (d) S 2p. 

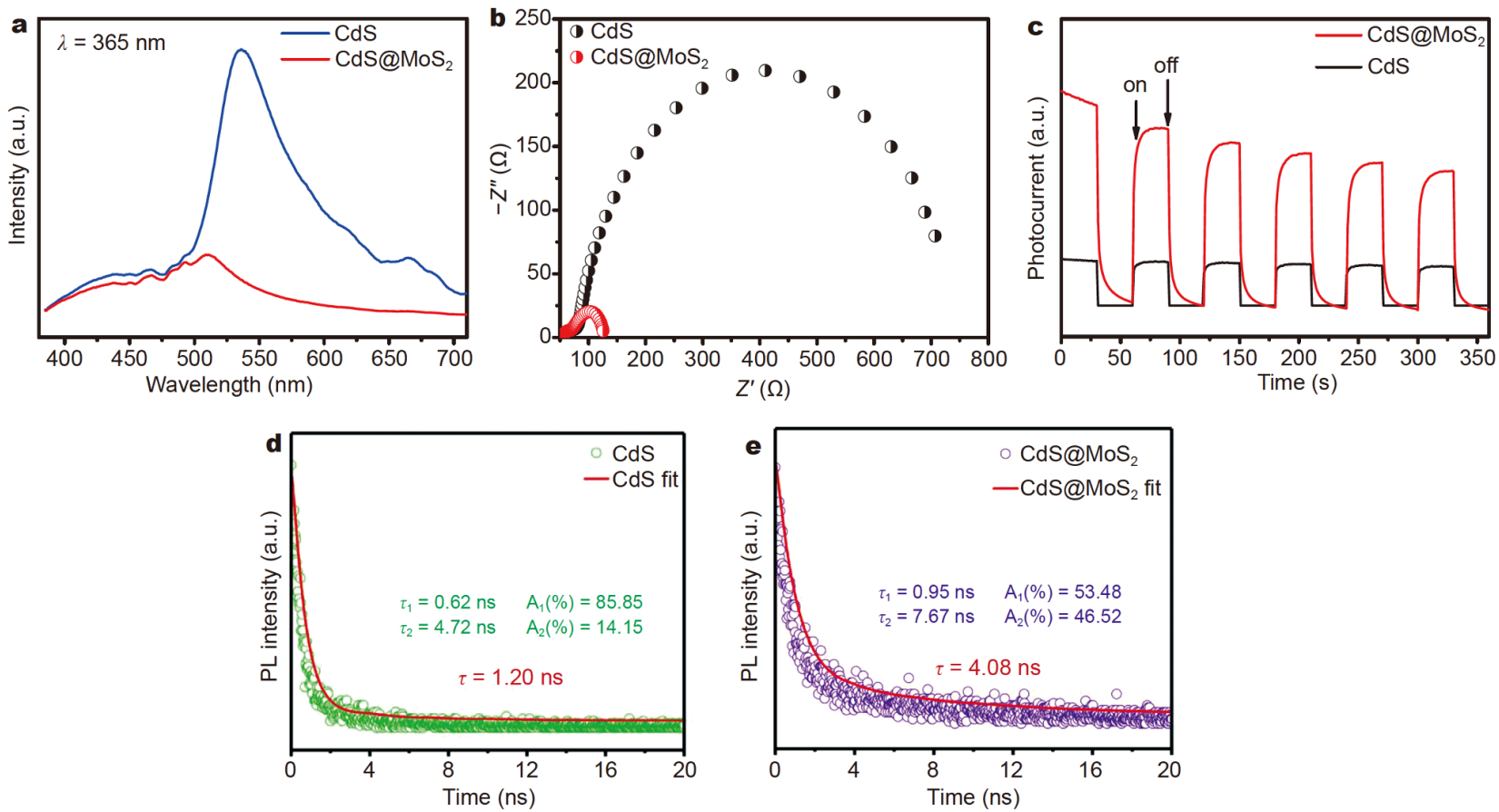

Figure 6 (a) PL spectra, (b) EIS Nyquist plots, (c) transient photocurrent responses under visible light irradiation $(\lambda>420 \mathrm{~nm})$, time-resolved PL spectra for CdS (d) and CdS@MoS 2 (e) photocatalysts.

CdS@MoS${ }_{2}$ photocatalyst, which results in a more effective separation of electron-hole pairs. Moreover, photocurrent measurements were also used to confirm the high efficiency of charge generation, separation, and transmission of the CdS@MoS 2 composite. As shown in Fig. 6c, the CdS@MoS ${ }_{2}$ coated onto FTO glass exhibited a higher photocurrent response than the bare CdS nanorods under irradiation, indicating that more electron-hole pairs were excited; these photo-excited electrons can be efficiently transferred to the active sites, thereby improving the photocatalytic activity. The recombination kinetics of photo-generated charge carriers of the photocatalyst were further studied by time-resolved fluorescence spectroscopy. CdS@MoS $\mathrm{M}_{2}$ presented an average PL lifetime of $4.08 \mathrm{~ns}$ (Fig. 6d), which is longer than that of bare CdS (1.20 ns, Fig. 6e). This is because $\mathrm{MoS}_{2}$ forms a p-n junction with CdS, which lengthens the lifetime of the charge [56,57]. Under visible light irradiation, the Zscheme CdS@MoS 2 catalyst produced electron-hole pairs. However, the holes on CdS were consumed by the electrons on $\mathrm{MoS}_{2}$, resulting in the slower recombination of electron-hole pairs. Thus, the lifetime was prolonged and the PL intensity of CdS@MoS 2 became weaker. Owing to a prolonged lifetime, the charge carrier lifetime and charge separation efficiency of CdS@MoS ${ }_{2}$ were enhanced and its photocatalytic performance was greatly improved

\section{$[15,21,30,58]$.}

The light absorption of CdS NRs, pure $\mathrm{MoS}_{2}$ and the $\mathrm{CdS} @ \mathrm{MoS}_{2}$ samples were explored by using UV-Vis diffuse reflectance spectroscopy (Fig. S4). The absorption spectrum of CdS@MoS 2 in the visible region was significantly improved compared with bare CdS nanorods. The bandgaps of CdS and $\mathrm{MoS}_{2}$ were obtained using UVVis spectra and measured as 2.30 and $1.80 \mathrm{eV}$, respectively (Fig. S5a and b). Mott-Schottky plots were used to determine the energy band positions of CdS and $\mathrm{MoS}_{2}$, as well as the type of semiconductor. The CdS showed a flat band potential $\left(E_{\mathrm{fb}}\right)$ of $-0.92 \mathrm{~V} v s . \mathrm{Ag} / \mathrm{AgCl}$, with a positive slope (Fig. S6a); this suggests an n-type semiconductor [59]. Similarly, the $E_{\mathrm{fb}}$ of $\mathrm{MoS}_{2}$ was $2.25 \mathrm{~V}$ vs. $\mathrm{Ag} / \mathrm{AgCl}$ and had a negative slope (Fig. S6b), indicating that it is a p-type material [60]. For n-type semiconductors, the $E_{\mathrm{fb}}$ is near the bottom of the $\mathrm{CB}$, while for p-type semiconductors, the $E_{\mathrm{fb}}$ is near the top of the VB [61]. Therefore, the CB of CdS and the VB of $\mathrm{MoS}_{2}$ are approximately -0.72 and $2.45 \mathrm{~V}$ ( $v$ s. normal hydrogen electrode, NHE), respectively. Combined with the bandgap measurement results, the positions of the $\mathrm{CB}$ and $\mathrm{VB}$ of CdS were determined to be -0.72 and $1.58 \mathrm{~V}$ (vs. NHE), respectively. Those of $\mathrm{MoS}_{2}$ were 0.65 and $2.45 \mathrm{~V}$, respectively.

To understand the benzyl alcohol dehydrogenation 
process over CdS@MoS 2 , the electron paramagnetic resonance (EPR) technique using phenyl- $N$-tert-butylnitrone $(\mathrm{PBN})$ as a trapping reagent was applied to monitor the reaction intermediates in-situ. Fig. 7a shows a typical EPR spectrum of the photocatalyst in acetonitrile suspension containing benzyl alcohol under anaerobic conditions. The result shows a triplet signal with relative intensities of 1:1:1 emerged over the CdS@MoS 2 composite when $\mathrm{PBN}$ was added to the reaction system under visible light irradiation. This can be due to the PBNcarbon centered radical (PBN-C) formed by $\cdot \mathrm{C}$ trapped on $\mathrm{PBN}$, wherein a triplet signal is split into six peaks, indicating that one corresponds to carbon radical and one $\mathrm{H}$ atom forms $\mathrm{C}-\mathrm{H}[62,63]$. This result is consistent with the mechanism of benzyl alcohol oxidation (Fig. 7b). All the above results indicate that $\mathrm{C}$ induced by the photogenerated holes are the key intermediates of the photocatalytic dehydrogenation of benzyl alcohol. However, no EPR signals were observed in the dark, indicating that the conversion process of $\mathrm{PhCH}_{2} \mathrm{OH}$ is indeed initiated by visible-light-driven photocatalysis. This result is consistent with the above test of photocatalytic activity.

The above analysis indicates that the improvement in photocatalytic activity is attributed to efficient charge transfer and separation. According to the traditional band-band transfer mechanism, photogenerated electrons transfer from CdS with a more negative CB to the $\mathrm{MoS}_{2}$, and photogenerated holes transfer from $\mathrm{MoS}_{2}$ with a more positive $\mathrm{VB}$ to the $\mathrm{CdS}$ (Fig. 7c). If so, the electrons accumulated in the $\mathrm{CB}$ of $\mathrm{MoS}_{2}$ cannot reduce $\mathrm{H}^{+}$to $\mathrm{H}_{2}$ $\left(\mathrm{H}^{+} / \mathrm{H}_{2}\right.$ vs. NHE, $\left.-0.41 \mathrm{~V}\right)$, and the holes accumulated in the VB of CdS cannot oxidize benzyl alcohol to benzaldehyde (BA/BAD vs. NHE, $1.98 \mathrm{~V}$ ), because this does not conform to the redox theory of reduction potential. However, the CdS@MoS${ }_{2}$ catalyst exhibited high activity for the production of hydrogen and benzaldehyde. In addition, when p-type $\mathrm{MoS}_{2}$ was incorporated into n-type $\mathrm{CdS}$, the electrons were transferred from the higher Fermi level to the lower Fermi level until the two Fermi levels reached the same Fermi level. As the Fermi level tends to be consistent $[57,64]$, the internal electric fields of p-type negative charge $\left(\mathrm{MoS}_{2}\right)$ and n-type positive charge (CdS) are formed, which is due to the accumulation and loss of electrons, respectively. This causes in the band to bend downward and upward. Interestingly, downward bending $\mathrm{MoS}_{2}$ and upward bending CdS provide a smooth channel for charge transport, allowing the electrons in the $\mathrm{MoS}_{2} \mathrm{CB}$ and the holes in the CdS VB to easily recombine
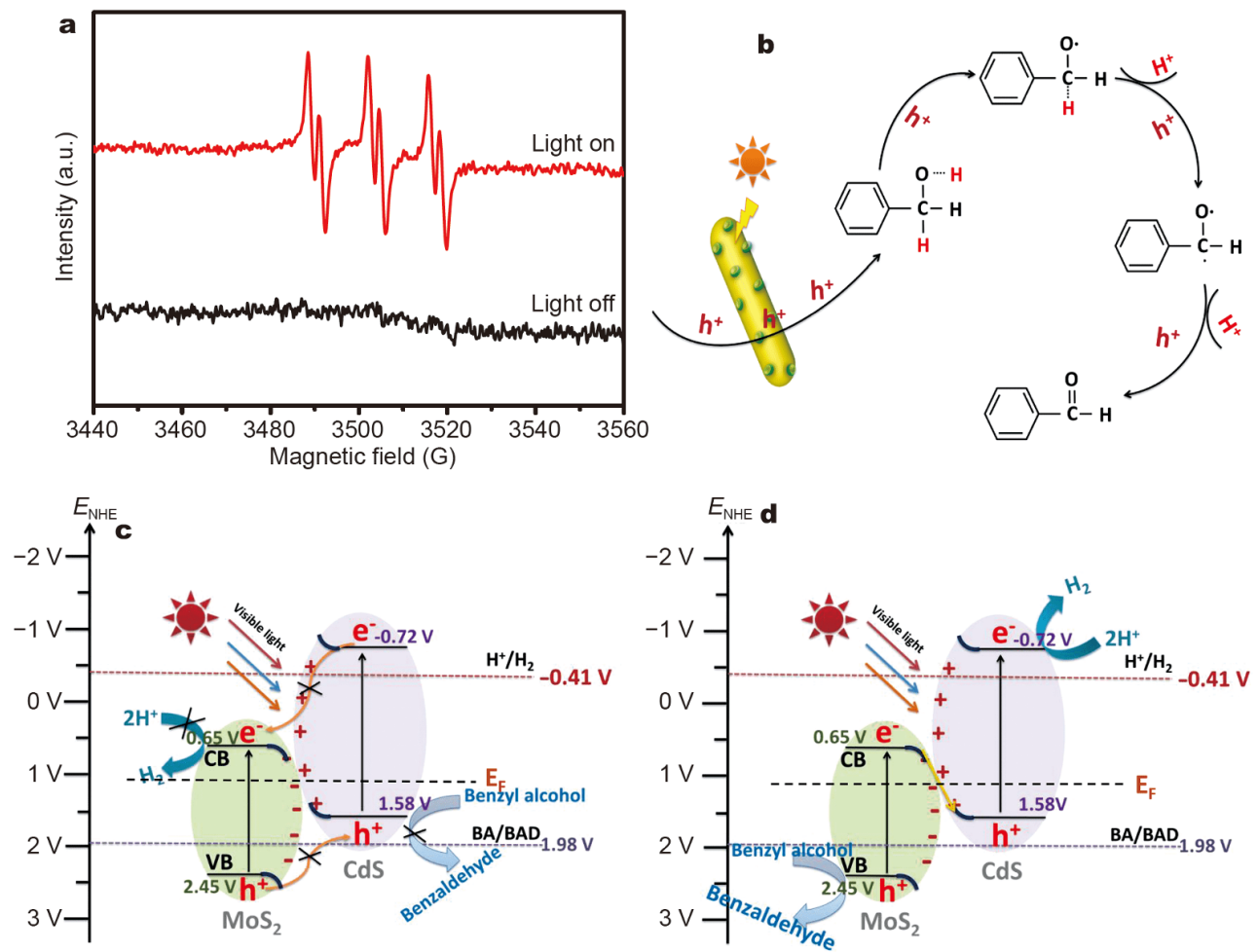

Figure 7 (a) EPR spectra of the PBN-carbon centered radical. (b) Mechanism of benzyl alcohol oxidation into benzaldehyde. (c) Band-band transfer mechanism and (d) Z-scheme transfer mechanism. 
[57]. In general, if the direction of the internal electric field is opposite to the direction of electron transfer, it is consistent with the band-band transfer mechanism; otherwise, it is a Z-scheme transfer [36,57,61]. Obviously, the direction of the electric field of the CdS@MoS system is directed from the n-type CdS with positive charge to the p-type $\mathrm{MoS}_{2}$ with negative charge, and is consistent with the electron transfer direction. Therefore, the Zscheme transfer mechanism is preferred for the CdS@MoS${ }_{2}$ heterojunction. As shown in Fig. 7d, the photogenerated electrons in the CB of $\mathrm{MoS}_{2}$ and the photogenerated holes in the $\mathrm{VB}$ of $\mathrm{CdS}$ rapidly recombined, thereby accumulating a large number of electrons in the $\mathrm{CB}$ of CdS and a large number of holes in the VB of $\mathrm{MoS}_{2}$. This provided a stronger redox capacity for hydrogen generation and benzyl alcohol oxidation. Moreover, the Z-scheme electron-hole transfer direction is consistent with the internal charge transfer direction of p-n junctions, which corresponds to the experimental results. Therefore, a possible reaction mechanism for the dehydrogenation of benzyl alcohol with the simultaneous production of hydrogen and benzaldehyde over CdS@MoS${ }_{2}$ heterostructures was proposed. Electron-hole pairs are generated under visible light excitation, and the electrons migrate to the $\mathrm{CB}$ position, leaving holes in the VB. Due to the Z-Scheme transfer mechanism, the electrons in the $\mathrm{CB}$ of p-type $\mathrm{MoS}_{2}$ will rapidly recombine with the holes in the $\mathrm{VB}$ of n-type CdS. A large number of holes in the VB of $\mathrm{MoS}_{2}$ can then oxidize benzyl alcohol into benzaldehyde (see Fig. $7 \mathrm{~b}$ ), and the $\mathrm{H}$ protons extracted from benzyl alcohol are reduced to $\mathrm{H}_{2}$ by the electrons accumulated in the $\mathrm{CB}$ of CdS. The Z-scheme mode of CdS@ $\mathrm{MoS}_{2}$ efficiently utilizes the high reduction capacity of CdS and the strong oxidation capacity of $\mathrm{MoS}_{2}$. The hole mobility of $\mathrm{MoS}_{2}$ is much greater than its electron mobility $[65,66]$, which is more conducive to the oxidation of benzyl alcohol on its surface. As seen from the UV-Vis spectra (Fig. S5) and the Mott-Schottky plots (Fig. S6), the VB of CdS was lower than the oxidation potential of benzyl alcohol, and cannot oxidize benzyl alcohol. However, the suitable VB position of $\mathrm{MoS}_{2}$ can effectively oxidize benzyl alcohol to benzaldehyde, indicating that the oxidation site is mainly on $\mathrm{MoS}_{2}$. In addition, the oxidation potential of benzyl alcohol into benzaldehyde was $1.98 \mathrm{~V} v s$. NHE, whereas the oxidation potential of benzaldehyde further oxidized was $2.5 \mathrm{~V} v s$. NHE [67]. However, the VB position of CdS@ $\mathrm{MoS}_{2}$ $(2.45 \mathrm{~V} v s . \mathrm{NHE})$ is exactly between them. Thus, benzyl alcohol is easily oxidized into benzaldehyde, and it is difficult for benzaldehyde to be further oxidized, resulting in the high selectivity of our study system. The introduction of $\mathrm{MoS}_{2}$ onto CdS nanorods significantly improved the dehydrogenation performance of benzyl alcohol. This was due to the formation of $\mathrm{p}-\mathrm{n}$ junctions between $\mathrm{MoS}_{2}$ and the CdS nanorods, exposing more active sites, and adopting a Z-scheme transfer mechanism, which boost the rapid separation of electron-hole pairs and increase the life of the carrier $[36,68,69]$.

\section{CONCLUSIONS}

In conclusion, we successfully realized the highly efficient, simultaneous production of hydrogen and the selective oxidation of benzyl alcohol into benzaldehyde over noble metal-free CdS@ $\mathrm{MoS}_{2}$ heterostructures under visible light $(\lambda>420 \mathrm{~nm})$. The CdS@MoS 2 photocatalyst demonstrated excellent hydrogen generation and high selectivity for benzaldehyde production, which was higher than that of bare CdS nanorods. The present study provides an effective $Z$-scheme charge transfer direction for directly utilizing photo-generated electrons and holes to achieve hydrogen production with simultaneous valueadded oxidation reactions without the need for noble metal catalysts or sacrificial agents.

Received 6 May 2020; accepted 1 July 2020;

published online 18 September 2020

1 Kudo A, Miseki Y. Heterogeneous photocatalyst materials for water splitting. Chem Soc Rev, 2009, 38: 253-278

$2 \mathrm{Li} \mathrm{X}, \mathrm{Yu}$ J, Low J, et al. Engineering heterogeneous semiconductors for solar water splitting. J Mater Chem A, 2015, 3: 2485-2534

3 Lu H, Zhao J, Li L, et al. Selective oxidation of sacrificial ethanol over $\mathrm{TiO}_{2}$-based photocatalysts during water splitting. Energy Environ Sci, 2011, 4: 3384

4 Xu HQ, Yang S, Ma X, et al. Unveiling charge-separation dynamics in CdS/Metal-organic framework composites for enhanced photocatalysis. ACS Catal, 2018, 8: 11615-11621

5 Chen X, Li C, Grätzel M, et al. Nanomaterials for renewable energy production and storage. Chem Soc Rev, 2012, 41: 7909-7937

6 Maeda $\mathrm{K}$, Teramura $\mathrm{K}, \mathrm{Lu} \mathrm{D}$, et al. Photocatalyst releasing hydrogen from water. Nature, 2006, 440: 295

7 Shen L, Luo M, Liu Y, et al. Noble-metal-free $\mathrm{MoS}_{2}$ co-catalyst decorated UiO-66/CdS hybrids for efficient photocatalytic $\mathrm{H}_{2}$ production. Appl Catal B-Environ, 2015, 166-167: 445-453

8 Turner JA. Sustainable hydrogen production. Science, 2004, 305: 972-974

9 Yang MQ, Zhang N, Pagliaro M, et al. Artificial photosynthesis over graphene-semiconductor composites. Are we getting better? Chem Soc Rev, 2014, 43: 8240-8254

10 Liu $\mathrm{H}, \mathrm{Xu} \mathrm{C}$, Li D, et al. Photocatalytic hydrogen production coupled with selective benzylamine oxidation over MOF composites. Angew Chem Int Ed, 2018, 57: 5379-5383

11 Kampouri S, Stylianou KC. Dual-functional photocatalysis for simultaneous hydrogen production and oxidation of organic substances. ACS Catal, 2019, 9: 4247-4270 
12 Li J, Li M, Sun H, et al. Understanding of the oxidation behavior of benzyl alcohol by peroxymonosulfate via carbon nanotubes activation. ACS Catal, 2020, 10: 3516-3525

13 Liu Y, Zhang P, Tian B, et al. Core-shell structural CdS@SnO nanorods with excellent visible-light photocatalytic activity for the selective oxidation of benzyl alcohol to benzaldehyde. ACS Appl Mater Interfaces, 2015, 7: 13849-13858

14 Yang $\mathrm{X}$, Zhao $\mathrm{H}$, Feng J, et al. Visible-light-driven selective oxidation of alcohols using a dye-sensitized $\mathrm{TiO}_{2}$-polyoxometalate catalyst. J Catal, 2017, 351: 59-66

15 Meng S, Ye X, Zhang J, et al. Effective use of photogenerated electrons and holes in a system: Photocatalytic selective oxidation of aromatic alcohols to aldehydes and hydrogen production. J Catal, 2018, 367: 159-170

16 Jiang $\mathrm{D}$, Chen $\mathrm{X}$, Zhang Z, et al. Highly efficient simultaneous hydrogen evolution and benzaldehyde production using cadmium sulfide nanorods decorated with small cobalt nanoparticles under visible light. J Catal, 2018, 357: 147-153

$17 \mathrm{Ni}$ M, Leung MKH, Leung DYC, et al. A review and recent developments in photocatalytic water-splitting using $\mathrm{TiO}_{2}$ for hydrogen production. Renew Sustain Energy Rev, 2007, 11: 401-425

18 Hao X, Wang Y, Zhou J, et al. Zinc vacancy-promoted photocatalytic activity and photostability of $\mathrm{ZnS}$ for efficient visiblelight-driven hydrogen evolution. Appl Catal B-Environ, 2018, 221: 302-311

19 Zhu C, Liu C, Zhou Y, et al. Carbon dots enhance the stability of CdS for visible-light-driven overall water splitting. Appl Catal BEnviron, 2017, 216: 114-121

$20 \mathrm{Xu} \mathrm{Y,} \mathrm{Zhao} \mathrm{W,} \mathrm{Xu} \mathrm{R,} \mathrm{et} \mathrm{al.} \mathrm{Synthesis} \mathrm{of} \mathrm{ultrathin} \mathrm{CdS} \mathrm{nanosheets}$ as efficient visible-light-driven water splitting photocatalysts for hydrogen evolution. Chem Commun, 2013, 49: 9803-9805

21 Reddy DA, Kim EH, Gopannagari M, et al. Enhanced photocatalytic hydrogen evolution by integrating dual co-catalysts on heterophase CdS nano-junctions. ACS Sustain Chem Eng, 2018, 6: 12835-12844

22 Liu Y, Zeng C, Ai L, et al. Boosting charge transfer and hydrogen evolution performance of CdS nanocrystals hybridized with $\mathrm{MoS}_{2}$ nanosheets under visible light irradiation. Appl Surf Sci, 2019, 484: 692-700

23 Xu Y, Huang Y, Zhang B. Rational design of semiconductor-based photocatalysts for advanced photocatalytic hydrogen production: the case of cadmium chalcogenides. Inorg Chem Front, 2016, 3: 591-615

24 Jiang W, Liu Y, Zong R, et al. Photocatalytic hydrogen generation on bifunctional ternary heterostructured $\mathrm{In}_{2} \mathrm{~S}_{3} / \mathrm{MoS}_{2} / \mathrm{CdS}$ composites with high activity and stability under visible light irradiation. J Mater Chem A, 2015, 3: 18406-18412

25 Tang ZR, Han B, Han C, et al. One dimensional CdS based materials for artificial photoredox reactions. J Mater Chem A, 2017, 5: 2387-2410

26 Zhang N, Liu S, Fu X, et al. Fabrication of coenocytic Pd@CdS nanocomposite as a visible light photocatalyst for selective transformation under mild conditions. J Mater Chem, 2012, 22: 50425052

27 Li YB, Li T, Dai XC, et al. Precise tuning of coordination positions for transition-metal ions via layer-by-layer assembly to enhance solar hydrogen production. ACS Appl Mater Interfaces, 2020, 12: 4373-4384

$28 \mathrm{Wu} \mathrm{K}$, Chen $\mathrm{Z}, \mathrm{Lv} \mathrm{H}$, et al. Hole removal rate limits photodriven $\mathrm{H}_{2}$ generation efficiency in CdS-Pt and CdSe/CdS-Pt semi- conductor nanorod-metal tip heterostructures. J Am Chem Soc, 2014, 136: 7708-7716

29 Liu Y, Yu YX, Zhang WD. $\mathrm{MoS}_{2} / \mathrm{CdS}$ Heterojunction with high photoelectrochemical activity for $\mathrm{H}_{2}$ evolution under visible light: The role of $\mathrm{MoS}_{2}$. J Phys Chem C, 2013, 117: 12949-12957

30 Zhou J, Zhang Z, Kong X, et al. A novel P-N heterojunction with staggered energy level based on $\mathrm{ZnFe}_{2} \mathrm{O}_{4}$ decorating $\mathrm{SnS}_{2}$ nanosheet for efficient photocatalytic degradation. Appl Surf Sci, 2020, 510: 145442

31 Liu Y, Niu H, Gu W, et al. In-situ construction of hierarchical CdS/ $\mathrm{MoS}_{2}$ microboxes for enhanced visible-light photocatalytic $\mathrm{H}_{2}$ production. Chem Eng J, 2018, 339: 117-124

32 Yin XL, Li LL, Liu ML, et al. $\mathrm{MoS}_{x} / \mathrm{CdS}$ nano-heterostructures accurately constructed on the defects of CdS for efficient photocatalytic $\mathrm{H}_{2}$ evolution under visible light irradiation. Chem Eng J, 2019, 370: 305-313

33 Liang G, Waqas M, Yang B, et al. Enhanced photocatalytic hydrogen evolution under visible light irradiation by p-type $\mathrm{MoS}_{2} / \mathrm{n}$ type $\mathrm{Ni}_{2} \mathrm{P}$ doped g- $\mathrm{C}_{3} \mathrm{~N}_{4}$. Appl Surf Sci, 2020, 504: 144448

34 Li Y, Wang L, Cai T, et al. Glucose-assisted synthesize 1D/2D nearly vertical CdS/ $\mathrm{MoS}_{2}$ heterostructures for efficient photocatalytic hydrogen evolution. Chem Eng J, 2017, 321: 366-374

35 Reddy DA, Park H, Ma R, et al. Heterostructured $\mathrm{WS}_{2}-\mathrm{MoS}_{2}$ ultrathin nanosheets integrated on CdS nanorods to promote charge separation and migration and improve solar-driven photocatalytic hydrogen evolution. ChemSusChem, 2017, 10: 1563-1570

36 Zheng W, Feng W, Zhang X, et al. Anisotropic growth of nonlayered CdS on $\mathrm{MoS}_{2}$ monolayer for functional vertical heterostructures. Adv Funct Mater, 2016, 26: 2648-2654

37 Niu X, Bai X, Zhou Z, et al. Rational design and characterization of direct Z-scheme photocatalyst for overall water splitting from excited state dynamics simulations. ACS Catal, 2020, 10: 1976-1983

38 Zhang L, Zhang $\mathrm{H}$, Jiang C, et al. Z-scheme system of $\mathrm{WO}_{3} @ \mathrm{MoS}_{2} /$ CdS for photocatalytic evolution $\mathrm{H}_{2}: \mathrm{MoS}_{2}$ as the charge transfer mode switcher, electron-hole mediator and cocatalyst. Appl Catal B-Environ, 2019, 259: 118073

39 Zhao H, Yang X, Xu R, et al. CdS/ $\mathrm{NH}_{2}-\mathrm{UiO}-66$ hybrid membrane reactors for the efficient photocatalytic conversion of $\mathrm{CO}_{2}$. J Mater Chem A, 2018, 6: 20152-20160

40 Wang H, Naghadeh SB, Li C, et al. Enhanced photoelectrochemical and photocatalytic activities of CdS nanowires by surface modification with $\mathrm{MoS}_{2}$ nanosheets. Sci China Mater, 2018, 61: 839850

41 Hao H, Zhang L, Wang W, et al. Photocatalytic hydrogen evolution coupled with efficient selective benzaldehyde production from benzyl alcohol aqueous solution over $\mathrm{ZnS}-\mathrm{Ni}_{x} \mathrm{~S}_{y}$ composites. ACS Sustain Chem Eng, 2019, 7: 10501-10508

42 Chai Z, Zeng TT, Li Q, et al. Efficient visible light-driven splitting of alcohols into hydrogen and corresponding carbonyl compounds over a Ni-modified CdS photocatalyst. J Am Chem Soc, 2016, 138: 10128-10131

43 Li F, Wang Y, Du J, et al. Simultaneous oxidation of alcohols and hydrogen evolution in a hybrid system under visible light irradiation. Appl Catal B-Environ, 2018, 225: 258-263

44 Zhang L, Jiang D, Irfan RM, et al. Highly efficient and selective photocatalytic dehydrogenation of benzyl alcohol for simultaneous hydrogen and benzaldehyde production over Ni-decorated $\mathrm{Zn}_{0.5} \mathrm{Cd}_{0.5} \mathrm{~S}$ solid solution. J Energy Chem, 2019, 30: 71-77

45 Hong S, Kumar DP, Kim EH, et al. Earth abundant transition metal-doped few-layered $\mathrm{MoS}_{2}$ nanosheets on CdS nanorods for 
ultra-efficient photocatalytic hydrogen production. J Mater Chem A, 2017, 5: 20851-20859

46 Li LL, Yin XL, Pang DH, et al. One-pot synthesis of $\mathrm{MoS}_{2} / \mathrm{CdS}$ nanosphere heterostructures for efficient $\mathrm{H}_{2}$ evolution under visible light irradiation. Int J Hydrogen Energy, 2019, 44: 31930-31939

47 Reddy DA, Park H, Hong S, et al. Hydrazine-assisted formation of ultrathin $\mathrm{MoS}_{2}$ nanosheets for enhancing their co-catalytic activity in photocatalytic hydrogen evolution. J Mater Chem A, 2017, 5: 6981-6991

48 Xie J, Zhang J, Li S, et al. Controllable disorder engineering in oxygen-incorporated $\mathrm{MoS}_{2}$ ultrathin nanosheets for efficient hydrogen evolution. J Am Chem Soc, 2013, 135: 17881-17888

49 Yi JD, Shi PC, Liang J, et al. Porous hollow $\mathrm{MoS}_{2}$ microspheres derived from core-shell sulfonated polystyrene microspheres@MoS${ }_{2}$ nanosheets for efficient electrocatalytic hydrogen evolution. Inorg Chem Front, 2017, 4: 741-747

50 Yan Z, Du L, Lee Phillips D. Multilayer core-shell $\mathrm{MoS}_{2} / \mathrm{CdS}$ nanorods with very high photocatalytic activity for hydrogen production under visible-light excitation and investigation of the photocatalytic mechanism by femtosecond transient absorption spectroscopy. RSC Adv, 2017, 7: 55993-55999

51 Yin XL, Li LL, Jiang WJ, et al. $\mathrm{MoS}_{2} / \mathrm{CdS}$ nanosheets-on-nanorod heterostructure for highly efficient photocatalytic $\mathrm{H}_{2}$ generation under visible light irradiation. ACS Appl Mater Interfaces, 2016, 8: 15258-15266

52 Hou $\mathrm{Y}$, Laursen $\mathrm{AB}$, Zhang J, et al. Layered nanojunctions for hydrogen-evolution catalysis. Angew Chem Int Ed, 2013, 52: 36213625

53 Kadam SR, Gosavi SW, Kale BB, et al. Unique CdS@MoS 2 core shell heterostructure for efficient hydrogen generation under natural sunlight. Sci Rep, 2019, 9: 12036

54 Liu H, Cheng S, Wu M, et al. Photoelectrocatalytic degradation of sulfosalicylic acid and its electrochemical impedance spectroscopy investigation. J Phys Chem A, 2000, 104: 7016-7020

55 Zhao X, Xu T, Yao W, et al. Photoelectrocatalytic degradation of 4chlorophenol at $\mathrm{Bi}_{2} \mathrm{WO}_{6}$ nanoflake film electrode under visible light irradiation. Appl Catal B-Environ, 2007, 72: 92-97

56 Zhao W, Li J, Dai B, et al. Simultaneous removal of tetracycline and $\mathrm{Cr}(\mathrm{VI})$ by a novel three-dimensional $\mathrm{AgI} / \mathrm{BiVO}_{4} \mathrm{p}$-n junction photocatalyst and insight into the photocatalytic mechanism. Chem Eng J, 2019, 369: 716-725

$57 \mathrm{Wu} \mathrm{H}$, Meng S, Zhang J, et al. Construction of two-dimensionally relative p-n heterojunction for efficient photocatalytic redox reactions under visible light. Appl Surf Sci, 2020, 505: 144638

58 Lin R, Wan J, Xiong $\mathrm{Y}$, et al. A quantitative study of charge carrier dynamics in well-defined $\mathrm{WO}_{3}$ nanowires and nanosheets: insight into the crystal facet effect in photocatalysis. J Am Chem Soc, 2018, 140: 9078-9082

59 Li J, Chen Y, Yang X, et al. Visible-light-mediated high-efficiency catalytic oxidation of sulfides using wrinkled $\mathrm{C}_{3} \mathrm{~N}_{4}$ nanosheets. J Catal, 2020, 381: 579-589

$60 \mathrm{Wu} \mathrm{Z}$, Ouyang M, Wang D. Construction of $\mathrm{WS}_{2} / \mathrm{MoSe}_{2}$ hetero- junction for efficient photoelectrocatalytic hydrogen evolution. Mater Sci Semicond Proc, 2020, 107: 104822

61 Chen C, Li M, Jia Y, et al. Surface defect-engineered silver silicate/ ceria $\mathrm{p}-\mathrm{n}$ heterojunctions with a flower-like structure for boosting visible light photocatalysis with mechanistic insight. J Colloid Interface Sci, 2020, 564: 442-453

62 Li H, Qin F, Yang Z, et al. New reaction pathway induced by plasmon for selective benzyl alcohol oxidation on $\mathrm{BiOCl}$ possessing oxygen vacancies. J Am Chem Soc, 2017, 139: 3513-3521

63 Sankar M, Nowicka E, Carter E, et al. The benzaldehyde oxidation paradox explained by the interception of peroxy radical by benzyl alcohol. Nat Commun, 2014, 5: 3332

64 Nouri M, Zare-Dehnavi N, Jamali-Sheini F, et al. Synthesis and characterization of type-II $\mathrm{p}\left(\mathrm{Cu}_{x} \mathrm{Se}_{y}\right) / \mathrm{n}\left(\mathrm{g}-\mathrm{C}_{3} \mathrm{~N}_{4}\right)$ heterojunction with enhanced visible-light photocatalytic performance for degradation of dye pollutants. Colloids Surfs A-Physicochem Eng Aspects, 2020, 595: 124656

65 Wang Y, Zhang Z, Zhang L, et al. Visible-Light driven overall conversion of $\mathrm{CO}_{2}$ and $\mathrm{H}_{2} \mathrm{O}$ to $\mathrm{CH}_{4}$ and $\mathrm{O}_{2}$ on 3D-SiC@2D-MoS heterostructure. J Am Chem Soc, 2018, 140: 14595-14598

$66 \mathrm{Xu} \mathrm{G}$, Wang X, Sun Y, et al. Metallic and ferromagnetic $\mathrm{MoS}_{2}$ nanobelts with vertically aligned edges. Nano Res, 2015, 8: 29462953

67 Xiao X, Jiang J, Zhang L. Selective oxidation of benzyl alcohol into benzaldehyde over semiconductors under visible light: The case of $\mathrm{Bi}_{12} \mathrm{O}_{17} \mathrm{Cl}_{2}$ nanobelts. Appl Catal B-Environ, 2013, 142-143: 487493

68 Yin XL, Liu J, Jiang WJ, et al. Urchin-like Au@CdS/ $\mathrm{WO}_{3}$ micro/ nano heterostructure as a visible-light driven photocatalyst for efficient hydrogen generation. Chem Commun, 2015, 51: 1384213845

69 Zhao Q, Ji M, Qian H, et al. Controlling structural symmetry of a hybrid nanostructure and its effect on efficient photocatalytic hydrogen evolution. Adv Mater, 2014, 26: 1387-1392

Acknowledgements This work was supported by the National Key Research and Development Program of China (2017YFA0700102), the National Natural Science Foundation of China (21520102001, 21871263 and 21671188), the Key Research Program of Frontier Sciences, CAS (QYZDJ-SSW-SLH045), and the Strategic Priority Research Program of the Chinese Academy of Sciences (XDB20000000).

Author contributions Gao S and Li P designed and engineered the experiments; Li P performed the experiments and the characterizations; Zhao $\mathrm{H}$ designed the materials; Li P wrote the paper with support from Gao $S$ and Cao R. All authors contributed to the general discussion.

Conflict of interest The authors declare that they have no conflict of interest.

Supplementary information Experimental details and supporting data are available in the online version of the paper. 


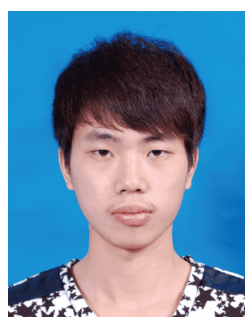

Peixian Li received his Bachelor's degree from the School of Materials Science and Engineering, Liaocheng University in 2018. He is currently a Master candidate jointly trained by Fujian Normal University and Fujian Institute of Research on the Structure of Matter (FJIRSM).

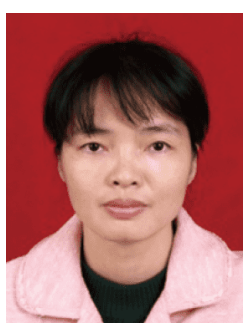

Shuiying Gao received her Master degree from Lanzhou Institute of Chemical Physics, Chinese Academy of Sciences (CAS) in 2002. She obtained her PhD from FJIRSM, CAS, in 2006. In 2014, she became a professor at FJIRSM. Her research interests focus on thin-film materials and photocatalysis.

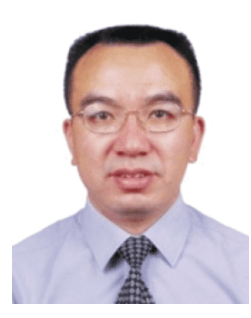

Rong Cao was born in Fujian Province, China. He obtained his $\mathrm{PhD}$ from FJIRSM, CAS, in 1993. Following post-doctoral experience at the Hong Kong Polytechnic University and a Japan Society for the Promotion of Science (JSPS) Fellowship at Nagoya University, he became a professor at FJIRSM in 1998. He is currently the director of FJIRSM. His main research interests include supramolecular chemistry, inorganicorganic hybrid materials, and nanocatalysis.

\section{$\mathrm{CdS} @ \mathrm{MoS}_{2}$ 异质结催化剂可见光光催化产氢和选 择性氧化苯甲醇的耦合反应}

李培贤 ${ }^{1,2,3,4}$, 赵慧 $1,3,4$, 间旭燕 ${ }^{1,2,3,4}$, 杨雪 ${ }^{1,3,4}$, 李静君 ${ }^{1,3,4}$,
高水英 $1,3,4^{*}$, 曹荣 ${ }^{1,3,4^{*}}$

摘要 光催化产氢, 并同时选择性氧化有机底物、生产高附加值精 细化学品, 引起了科学家的广泛关注. 在本文中, 我们报道了一种无 贵金属的 $\mathrm{CdS} @ \mathrm{MoS}_{2}$ 异质结光催化剂, 在可见光下可以高效地产氢 并同时将苯甲醇选择性氧化为苯甲醛. 在 $0.3 \mathrm{mmol}$ 苯甲醇底物的条 件下, CdS@ $\mathrm{MoS}_{2}$ 能够产生 $4233 \mu \mathrm{mol} \mathrm{g}^{-1} \mathrm{~h}^{-1}$ 的氢气, 比纯的CdS纳 米棒高约 53 倍, 并且苯甲醇选择性氧化为苯甲醛具有很高的选择 性; 当苯甲醇增加到 $1.0 \mathrm{mmol}$ 时, 产生氢气的量高达 $9033 \mu \mathrm{mol} \mathrm{g}{ }^{-1} \mathrm{~h}^{-1}$. 通过扫描电子显微镜和透射电子显微镜图像表 征, 发现 $\mathrm{p}$ 型 $\mathrm{MoS}_{2}$ 具有花状结构并紧密粘附在 $\mathrm{n}$ 型 $\mathrm{CdS}$ 半导体纳米 棒上, 从而形成 $\mathrm{p}-\mathrm{n}$ 异质结. 作为潜在的 Z型光催化剂, $\mathrm{CdS} @ \mathrm{MoS}_{2}$ 异质结催化剂在可见光下有效地产生和分离电子-空穴对, 其中电 子用于还原以产生氢气, 而空穴则将苯甲醇氧化为苯甲醛. 此外, 我们提出了光生电荷转移和分离的机理, 并通过苂光光谱、电化 学阻抗谱、光电流和莫特-肖特基测量进行了验证. 结果表明, $\mathrm{CdS} @ \mathrm{MoS}_{2}$ 异质结具有快速的电荷分离和转移效率, 极大地提高了 苯甲醇的脱氢性能. 这项工作为高性能Z型光催化剂的合理设计, 以及利用空穴和电子同时获得两种有价值的化学物质提供了思路. 